\title{
Exploratory Analysis Comparing Fosnetupitant Versus Fosaprepitant for Prevention of Highly Emetogenic Chemotherapy-Induced Nausea and Vomiting (CINV): A Randomized, Double-Blind, Phase 3 Study (CONSOLE)
}

\author{
Akito Hata $\cdot$ Yoshimasa Shiraishi · Naoki Inui · Morihito Okada • Masahiro Morise • \\ Kohei Akiyoshi · Masayuki Takeda · Yasutaka Watanabe · Shunichi Sugawara • \\ Naofumi Shinagawa $\cdot$ Kaoru Kubota $\cdot$ Toshiaki Saeki $\cdot$ Tomohide Tamura
}

Received: December 20, 2021 / Accepted: January 25, 2022 / Published online: March 4, 2022

(C) The Author(s) 2022, corrected publication 2022

\section{ABSTRACT}

Introduction: We describe the results of an exploratory analysis performed on the first head-to-head study (JapicCTI-194611) comparing two different intravenous (IV) neurokinin 1

Supplementary Information The online version contains supplementary material available at https://doi. org/10.1007/s40487-022-00188-2.

A. Hata $(\bowtie)$

Department of Thoracic Oncology, Kobe Minimally Invasive Cancer Center, 8-5-1 Minatojima-

minamimachi, Chuo-ku, Kobe, Hyogo 650-0047, Japan

e-mail: akitohata@hotmail.com

Y. Shiraishi

Research Institute for Diseases of the Chest, Graduate School of Medical Sciences, Kyushu University, Fukuoka, Japan

$\mathrm{N}$. Inui

Second Division, Department of Internal Medicine, Hamamatsu University School of Medicine,

Hamamatsu, Japan

M. Okada

Department of Surgical Oncology, Hiroshima

University, Hiroshima, Japan

\section{Morise}

Department of Respiratory Medicine, Nagoya University Graduate School of Medicine, Nagoya, Japan
$\left(\mathrm{NK}_{1}\right)$ receptor antagonists, fosnetupitant and fosaprepitant, in combination with palonosetron (PALO) and dexamethasone (DEX) for the prevention of highly emetogenic chemotherapy (HEC)-induced nausea and vomiting (CINV). This analysis was performed to validate the findings of the primary analysis (previously published) utilizing a last observation carried forward (LOCF) approach for missing values for the efficacy endpoint of complete response (no

\section{K. Akiyoshi \\ Department of Clinical Oncology, Osaka City \\ General Hospital, Osaka, Japan}

\section{Takeda}

Department of Medical Oncology, Kindai University Faculty of Medicine, Osaka-sayama, Japan

\section{Y. Watanabe}

Department of Thoracic Oncology, Saitama Cancer Center, Saitama, Japan

\section{S. Sugawara}

Department of Pulmonary Medicine, Sendai Kousei Hospital, Sendai, Japan

\section{N. Shinagawa}

Department of Respiratory Medicine, Faculty of Medicine and Graduate School of Medicine,

Hokkaido University, Sapporo, Japan

\section{K. Kubota}

Department of Pulmonary Medicine and Oncology, Graduate School of Medicine, Nippon Medical School, Tokyo, Japan 
emetic event and no rescue medication), while also evaluating the time periods encompassing the 0-168-hour (h) "extended overall phase" interval.

Methods: Patients scheduled to receive cisplatin-based chemotherapy were randomized 1:1 to fosnetupitant $235 \mathrm{mg}$ or fosaprepitant $150 \mathrm{mg}$ in combination with PALO $0.75 \mathrm{mg}$ and DEX. Complete response rates were calculated and compared (stratified by age category and sex with a Mantel-Haenszel test) during the study's primary overall phase $(0-120 \mathrm{~h})$ and during additional time intervals of interest [acute $(0-24 \mathrm{~h})$, delayed (24-120 h), extended delayed $(>24-168 \mathrm{~h})$, beyond delayed (120-168 h), and extended overall (0-168 h)].

Results: A total of 785 patients were included (fosnetupitant $N=392$, fosaprepitant $N=393$ ). Complete response rates were numerically higher for fosnetupitant versus fosaprepitant for all time intervals and statistically significant for the extended overall phase. Complete response rates for fosnetupitant versus fosaprepitant during the overall, acute, delayed, extended delayed, beyond delayed, and extended overall phases were $75.5 \%$ vs. $71.0 \%(p=0.1530), \quad 93.9 \%$ vs. $92.6 \% \quad(p=$ $0.4832), 77.0 \%$ vs. $72.8 \%(p=0.1682), 74.7 \%$ vs. $68.4 \%(p=0.0506), 86.7 \%$ vs. $81.7 \%(p=$ $0.0523)$, and $73.5 \%$ vs. $66.9 \%(p=0.0450)$, respectively.

Conclusion: In this exploratory analysis, fosnetupitant appeared to be more effective than fosaprepitant in preventing CINV associated with cisplatin-based HEC during the extended 7-day period following chemotherapy.

Keywords: Antiemetic; Aprepitant; CINV; Fosaprepitant; Fosnetupitant; NEPA; Palonosetron

T. Saeki

Department of Breast Oncology, Saitama Medical University International Medical Center, Saitama, Japan

T. Tamura

Thoracic Center, St. Luke's International Hospital, Tokyo, Japan

\section{Key Summary Points}

Chemotherapy-induced nausea and vomiting (CINV) is described as occurring in two arbitrarily defined phases: the acute phase (from 0 to $24 \mathrm{~h}$ following chemotherapy initiation) and the delayed phase (from $24 \mathrm{~h}$ to $120 \mathrm{~h}$ ), with almost all antiemetic studies evaluating treatment efficacy only to $120 \mathrm{~h}$ after the administration of chemotherapy.

This was the first study to compare two neurokinin $1\left(\mathrm{NK}_{1}\right)$ receptor antagonist (RA)-containing (fosnetupitant vs. fosaprepitant) antiemetic regimens beyond the $120 \mathrm{~h}$ time point for prevention of cisplatin-based highly emetogenic CINV.

The primary analysis of this study (previously published) showed noninferiority of fosnetupitant and fosaprepitant for overall 0-120 h complete response (no emetic event and no rescue medication) rates.

This exploratory analysis utilized a last observation carried forward (LOCF) approach for missing values for the complete response evaluation.

Complete response rates were numerically higher for fosnetupitant than fosaprepitant during all time intervals and significantly higher for fosnetupitant in the extended overall 0-168 h phase.

It may be prudent for clinicians to continue to assess these symptoms beyond the traditional 5-day period after cisplatin-based chemotherapy.

DIGITAL FEATURES This article is published with digital features, including an infographic, to facilitate understanding of the article. To view digital features for this article go to https:// doi.org/10.6084/m9.figshare.19494743. 


\section{INTRODUCTION}

Chemotherapy-induced nausea and vomiting (CINV) is a common distressing side effect associated with many chemotherapeutic drugs and regimens [1-4]. Not only can CINV have a detrimental effect on patients' quality of life [5], but it can also lead to chemotherapy dose reductions or discontinuation [6], thereby negatively impacting a patient's response to treatment.

Advances over the past three decades have not only helped elucidate mechanisms by which chemotherapeutic agents induce nausea and vomiting, but the American Society of Clinical Oncology (ASCO) has recognized the development of effective antiemetic therapies among the top five advances in oncology over the last 50 years [7]. Several neurotransmitters, including dopamine, serotonin (5-HT), and substance $P$, are now well established as important mediators of CINV [7]. The emetic response to chemotherapy is now thought to occur through both a peripheral and a central pathway $[8,9]$. The peripheral pathway, activated within 24 hours (h) following chemotherapy, is primarily associated with acute CINV, and involves the activation of the 5-hydroxytryptamine-3 (5-HT3) receptors in the vagal afferents that transmit the stimulus to the brain after enterochromaffin cells release 5-HT. The central pathway, mainly located in the brain, is activated $>24 \mathrm{~h}$ after chemotherapy initiation, and is primarily associated with delayed CINV; substance $\mathrm{P}$ is the principal neurotransmitter that activates neurokinin 1 (NK1) receptors in the central nervous system.

As the single most emetogenic chemotherapeutic and because of the predictability of emesis in virtually all patients after its use, cisplatin has served as the standard emetic stimulus for clinical trials of antiemetic drugs $[10,11]$. High-dose cisplatin induces a biphasic pattern of emesis; following an initial peak which occurs between 6 and $8 \mathrm{~h}$ after cisplatin infusion, there is a reduction in symptoms with a subsequent second phase of nausea and emesis, peaking between 24 and $72 \mathrm{~h}$, although symptoms may occur for several more days $[10,11]$.
Due to this biphasic time course of emesis following cisplatin, CINV is described as occurring in two arbitrarily defined phases [10]: the acute phase (from 0 to $24 \mathrm{~h}$ following chemotherapy initiation) and the delayed phase (from 24 to $120 \mathrm{~h}$ ). Almost all antiemetic studies have therefore evaluated efficacy to $120 \mathrm{~h}$ after the administration of chemotherapy.

While the period beyond $120 \mathrm{~h}$ (and between chemotherapy cycles) is generally perceived to be relatively free of CINV, studies have shown that nausea, in particular, is still present beyond day 5 for a substantial proportion of patients. Bossi and colleagues reported the results of cisplatin-based study data on ginger where $\sim 60-68 \%$ of patients had at least mild nausea ( $>5 \mathrm{~mm}$ on a $100 \mathrm{~mm}$ visual analog scale [VAS]) between cycles 1 and 2, and $30-42 \%$ had significant nausea ( $>25 \mathrm{~mm}$ VAS) [12]. Similarly, in another cisplatin study, Pessi et al. [13] showed that $10-27 \%$ of patients had at least mild nausea on days 14-16 of cycles 1,2 , and 3 , and a substantial proportion of patients (42-53\%) were taking rescue medication during this intercycle time period, despite receiving antiemetics according to guidelines [14].

NEPA is a unique fixed antiemetic combination comprising a highly selective $\mathrm{NK}_{1}$ receptor antagonist (RA; oral netupitant $300 \mathrm{mg}$ or intravenous fosnetupitant $235 \mathrm{mg}$ ) and a $5-\mathrm{HT}_{3}$ RA (palonosetron, PALO). The long elimination half-lives of oral netupitant and PALO could contribute to long-lasting CINV protection [15]. Prior studies evaluating oral NEPA in the cisplatin setting have shown better prevention of CINV with single-dose NEPA than a 3-day aprepitant regimen in the latter part of the delayed phase, specifically during days 3-5 following chemotherapy [16]. Oral and intravenous NEPA have been approved in the United States and the European Union. In Japan, approaches have focused on the development of intravenous fosnetupitant as a single agent.

The CONSOLE study (JapicCTI-194611) is the first study comparing two $\mathrm{NK}_{1} \mathrm{RA}$-containing antiemetic regimens beyond the $120 \mathrm{~h}$ time point. This was a non-inferiority study designed to assess the comparative efficacy of the intravenous $\mathrm{NK}_{1} \mathrm{RAs}$, fosnetupitant and fosaprepitant, both administered in combination with 
the 5- $\mathrm{HT}_{3} \mathrm{RA}, \mathrm{PALO}$, and dexamethasone (DEX) for the prevention of cisplatin-based highly emetogenic CINV. In this study antiemetic efficacy was assessed up to $168 \mathrm{~h}$, as a phase 2 study indicated an efficacy benefit of fosnetupitant beyond $120 \mathrm{~h}$ [17].

The primary analysis of the CONSOLE study has been published [18]. Non-inferiority of fosnetupitant and fosaprepitant was demonstrated for the primary endpoint of overall $(0-120 \mathrm{~h})$ complete response (no emetic event and no rescue medication). The overall complete response rate was $75.2 \%$ vs. $71.0 \%$, respectively (Mantel-Haenszel common risk difference $4.1 \%$; $95 \%$ confidence interval $-2.1 \%$ to $10.3 \%)$.

In this brief report, we describe the results of an exploratory post hoc analysis performed for this $\mathrm{NK}_{1} \mathrm{RA}$ comparative study. The intent of this analysis was to validate the findings of the primary analysis utilizing a last observation carried forward (LOCF) approach for missing values for the complete response evaluation, while also further evaluating the time periods encompassing the $0-168 \mathrm{~h}$ "extended overall phase" interval.

\section{METHODS}

\section{Study Design and Treatments}

This was a randomized, multicenter, doubleblind, double-dummy phase 3 study in patients receiving cisplatin-based chemotherapy conducted at 82 study sites in Japan. The primary endpoint of this study was to demonstrate the non-inferiority of fosnetupitant to fosaprepitant in terms of overall complete response rate in the first cycle. Patients who continued into the multiple cycle extension phase of the study received only the fosnetupitant regimen, with safety as the primary assessment.

This study was conducted according to the ethical principles of the Declaration of Helsinki and Good Clinical Practice guidelines, and was initiated at the participating study sites only after institutional review board approval.

Eligible patients were enrolled by investigators via an interactive web response system and were randomized 1:1 to either fosnetupitant or fosaprepitant treatment according to dynamic allocation per stratification factors (age category [ $\geq 55$ years vs. $<55$ years], sex, and study site). On day 1 (the study drug administration day) in the fosnetupitant group, fosnetupitant $235 \mathrm{mg}$, PALO $0.75 \mathrm{mg}$, and DEX $9.9 \mathrm{mg}$ were mixed together and infused for $30 \mathrm{~min}$, starting $1 \mathrm{~h}$ before the start of cisplatin administration. In the fosaprepitant group, fosaprepitant $150 \mathrm{mg}$ was infused for $30 \mathrm{~min}$, starting $1 \mathrm{~h}$ before the start of cisplatin administration, and PALO $0.75 \mathrm{mg}$ and DEX $9.9 \mathrm{mg}$ were separately infused for $30 \mathrm{~min}$, due to a potential risk of incompatibility of fosaprepitant with PALO, starting either $30 \mathrm{~min}$ before or immediately following fosaprepitant administration. On days 2-4, DEX $6.6 \mathrm{mg}$ was administered intravenously in both groups. To maintain blinding, physiological saline was administered in the fosnetupitant group at the same time PALO and DEX were to be administered in the fosaprepitant group. The study drugs were administered through peripheral veins. Patients were hospitalized from day 1 to day 8 .

\section{Patients}

Inclusion/exclusion criteria are presented in detail in the primary publication of this study [18]. Key eligibility criteria were that patients were $\geq 20$ years, naïve to chemotherapy or had received prior chemotherapy classified as low/ minimal emetic risk, and scheduled to receive cisplatin-based ( $\geq 70 \mathrm{mg} / \mathrm{m}^{2}$ ) chemotherapy for the treatment of a confirmed solid tumor malignancy. Patients were required to have an Eastern Cooperative Oncology Group (ECOG) performance status of $0-1$.

\section{Endpoints and Assessments}

During the $0-168 \mathrm{~h}$ (7-day) period postchemotherapy, each patient completed a diary, capturing emetic episodes and severity of nausea (4-point Likert scale) every $24 \mathrm{~h}$ from the time of cisplatin administration. Rescue medication was administered during this time interval if the investigator judged it necessary. 
Administration of rescue medication specifically for nausea was to be considered by investigators only when the nausea was "severe (with inability to eat and drink)."

The proportion of patients with complete response (no emetic event and no rescue medication) during the overall $(0-120 \mathrm{~h})$ phase was the primary endpoint in the study. Complete response rates were calculated during the overall phase and during additional time intervals of interest [i.e., acute (0-24 h), delayed (24-120 h), extended delayed $(>24-168 \mathrm{~h})$, beyond delayed (120-168 h), and extended overall (0-168 h)].

\section{Statistical Analysis}

For the primary analysis of the primary endpoint in this study [18], differences in the overall $0-120 \mathrm{~h}$ complete response rate were analyzed by comparing the complete response rates of fosnetupitant with that of fosaprepitant stratified by age category and sex and calculated on the basis of the Mantel-Haenszel method in the full analysis set (FAS; which includes only patients who received the study drug, PALO, DEX, and cisplatin on day 1). A two-sided 95\% confidence interval (CI) was also calculated using Newcombe's method. If the lower confidence bound was higher than $-10 \%$ (non-inferiority margin), non-inferiority was considered to be confirmed, and if it was higher than $0 \%$, superiority was also considered to be confirmed.

This exploratory analysis did not evaluate non-inferiority between treatments but rather only assessed differences in the complete response rates for fosnetupitant versus fosaprepitant, also stratified by age category and sex with a Mantel-Haenszel test.

The original analysis and the exploratory analysis differed in how missing data were handled. This exploratory analysis was performed on the FAS, but in this case a LOCF approach was utilized for missing values for complete response evaluation. To clarify, if there was no evidence of treatment failure (i.e., no emetic event and no use of rescue medication) for the duration of participation, the patient would be considered a complete responder at all time points following discontinuation or in the case of missing data. Similarly, if the last data reflected emetic event or use of rescue medication, the patient would be considered a treatment failure at all time points following discontinuation or in the case of missing data. Contrary to this LOCF approach, the original analysis on the FAS considered any patient who did not complete the study as a treatment failure. In addition, the purpose of the original analysis [18] was to test for both non-inferiority and superiority between the two arms, whereas the null hypothesis in this analysis was that there was no difference in the proportion of patients with complete response between the two arms. So, in addition to differences in the approach to handling missing values, the null hypotheses between the original study analysis and this one were also different.

\section{RESULTS}

\section{Patients and Baseline Characteristics}

As was the case with the original analysis, this exploratory analysis included a total of 785 patients $(N=392$ fosnetupitant and $N=393$ fosaprepitant). There were three patients with missing data during the efficacy observation period. One patient (fosnetupitant) was considered a treatment failure in both the original analysis and the LOCF analysis, while the other two patients (one fosnetupitant and one fosaprepitant) were reclassified from a treatment failure in the original analysis to a complete responder in the current LOCF exploratory analysis during at least one of the time intervals assessed (Supplementary Material).

Patient background characteristics are presented in Table 1 . The patient characteristics were generally similar between the two groups; most patients were male, and the predominant cancer type was lung cancer.

\section{Efficacy}

In this exploratory analysis, complete response rates were similar for fosnetupitant and 
Table 1 Patient characteristics

\begin{tabular}{|c|c|c|}
\hline & $\begin{array}{l}\text { Fosnetupitant } \\
(N=392)\end{array}$ & $\begin{array}{l}\text { Fosaprepitant } \\
(N=393)\end{array}$ \\
\hline \multicolumn{3}{|l|}{ Age } \\
\hline Median (range) & $67.0(40-81)$ & $66.0(33-82)$ \\
\hline \multicolumn{3}{|l|}{ Age $[\mathrm{n}(\%)]$} \\
\hline$<55$ years & $50(12.8)$ & $50(12.7)$ \\
\hline$\geq 55$ years & $342(87.2)$ & $343(87.3)$ \\
\hline \multicolumn{3}{|l|}{$\operatorname{Sex}[\mathbf{n}(\%)]$} \\
\hline Male & $301(76.8)$ & $302(76.8)$ \\
\hline Female & $91(23.2)$ & $91(23.2)$ \\
\hline \multicolumn{3}{|l|}{$\begin{array}{l}\text { Cancer type [n } \\
(\%)]\end{array}$} \\
\hline Lung & $353(90.1)$ & $341(86.8)$ \\
\hline Esophagus & $21(5.4)$ & $24(6.1)$ \\
\hline Head and neck & $7(1.8)$ & $10(2.5)$ \\
\hline Other & $11(2.8)$ & $18(4.6)$ \\
\hline \multicolumn{3}{|l|}{$\begin{array}{l}\text { Dose of } \\
\text { cisplatin (mg/ } \\
\left.\mathbf{m}^{2}\right)[\mathbf{n}(\%)]\end{array}$} \\
\hline$\geq 70$ to $<80$ & $144(36.7)$ & $165(42.0)$ \\
\hline$\geq 80$ to $<90$ & $243(62.0)$ & $221(56.2)$ \\
\hline$\geq 90$ & $5(1.3)$ & $7(1.8)$ \\
\hline
\end{tabular}

fosaprepitant during the acute, delayed, and overall phases (Fig. 1). The overall complete response rate was $75.5 \%$ vs. $71.0 \%$ in the fosnetupitant vs. the fosaprepitant group, respectively.

For the time intervals which extend beyond $120 \mathrm{~h}$, a significantly higher $(p=0.0450$, Mantel-Haenszel test) complete response rate was seen for fosnetupitant $(73.5 \%)$ than fosaprepitant $(66.9 \%)$ during the extended overall phase $(0-168 \mathrm{~h})$; the complete response rates during the extended delayed phase $(>24-168 \mathrm{~h}$ ) were $74.7 \%$ and $68.4 \%(p=0.0506)$ for fosnetupitant and fosaprepitant, respectively, and during the beyond delayed phase (120-168 h) were $86.7 \%$ and $81.7 \%(p=0.0523)$ for fosnetupitant and fosaprepitant, respectively (Fig. 1).

\section{DISCUSSION}

In the full publication of this study, we reported the non-inferiority of fosnetupitant to fosaprepitant for the primary endpoint of complete response during the overall $0-120 \mathrm{~h}$ phase [fosnetupitant $75.2 \%$ vs. fosaprepitant $71.0 \%$, difference of $4.1 \%$ (95\% CI $-2.1 \%$ to $10.3 \%)$ ] [18]. All secondary efficacy endpoints/time intervals of delayed, beyond delayed, and extended overall phases tended to be higher with fosnetupitant than with fosaprepitant.

Contrary to the original analysis which viewed any patient with missing data as a treatment failure, in this paper we present the results of an exploratory analysis utilizing the LOCF approach for missing data related to complete response. This analysis was intended not only to assess the consistency of the findings with the original analysis of the primary endpoint of overall complete response, but also to evaluate the comparative results between fosnetupitant and fosaprepitant for additional time intervals, inclusive of days $6-7$ following chemotherapy (i.e., 120-168 h).

Since establishing the classification of the acute and delayed phases correlating with the time course of emesis following cisplatin-based chemotherapy, antiemetic trials have consistently evaluated the efficacy of agents up to $120 \mathrm{~h}$ post-dose. To our knowledge this is the first study to evaluate two $\mathrm{NK}_{1}$ RA-containing regimens beyond $120 \mathrm{~h}$.

Complete response rates in this exploratory analysis during the overall phase $(75.5 \%$ vs. $71.0 \%$ for fosnetupitant and fosaprepitant, respectively, $p=0.1530$ ) were similar to the outcome of the primary original analysis in the FAS population. Similar complete response rates were also seen for fosnetupitant and fosaprepitant during the acute and delayed phases. However, while the findings in the original analysis revealed numerically higher complete response rates for the intervals containing the extended period up to $168 \mathrm{~h}$ [18], in this analysis using the LOCF approach a significantly higher complete response rate was seen for the fosnetupitant regimen compared with the fosaprepitant regimen during the extended overall 


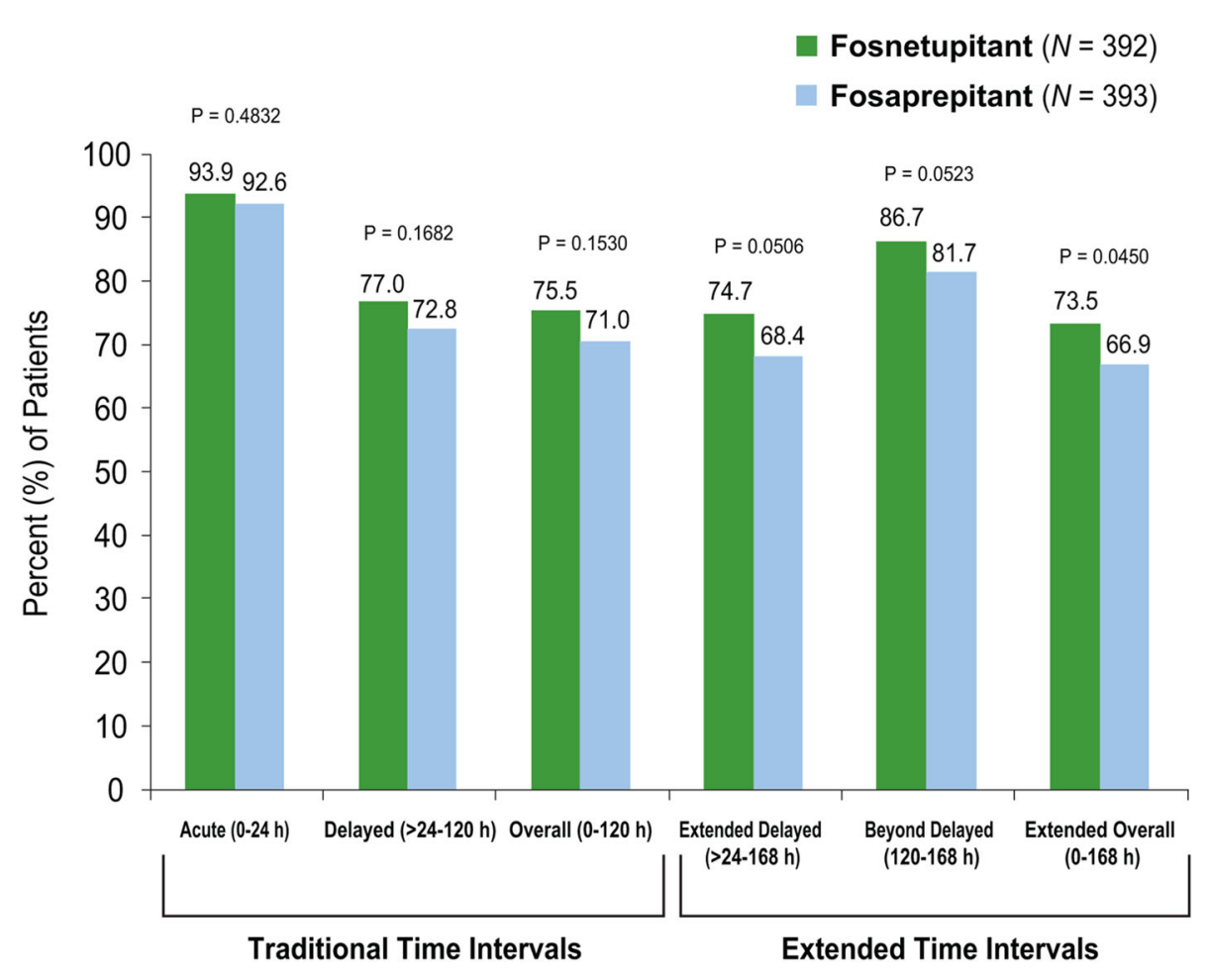

Fig. 1 Complete response rates during the traditional and extended time intervals: exploratory analysis

0-168 h phase; in addition, numerically higher complete response rates were also seen for fosnetupitant during the extended delayed (> 24-168 h) and beyond delayed (120-168 h) phases.

Conceivably, the differences in plasma elimination half-lives of the active form of these agents may offer an explanation for these differences. Fosnetupitant and fosaprepitant are rapidly converted to netupitant and aprepitant. The half-life of netupitant following a $30 \mathrm{~min}$ infusion is $144 \mathrm{~h}$ [15] compared with $9-13 \mathrm{~h}$ for aprepitant [19].

We reported a good safety profile for fosnetupitant in this study, with treatment-related adverse events consistent with those previously reported for $\mathrm{NK}_{1}$ RAs. Impressively, fosnetupitant was associated with significantly fewer injection site reactions (ISRs) than fosaprepitant in this study $(11.0 \%$ vs. $20.6 \%, p<0.001$ for any ISR, and $0.3 \%$ vs. $3.6 \%, p<0.001$ for treatment-related ISRs) [18].
It is noteworthy that a pooled analysis of three cisplatin registration studies for oral NEPA (fixed combination of netupitant and PALO) revealed significant differences favoring NEPA $(N=621)$ versus an oral aprepitant regimen $(N=576)$ during the delayed phase $(24-120 \mathrm{~h})$ for endpoints of complete response, complete protection, and no significant nausea [16]. In the subset of patients $(N=758)$ taking the $\geq 70 \mathrm{mg} / \mathrm{m}^{2}$ cisplatin (the dose in the current study), NEPA was superior to the aprepitant regimen for all efficacy endpoints during the delayed phase and more specifically for all endpoints during the individual days 3-5 postchemotherapy, suggesting that the efficacy of NEPA is long-lasting and sustained over time [16].

Based on the results of the current analysis and the few trials that have explored CINV control beyond $120 \mathrm{~h}$ following chemotherapy, it may be prudent for clinicians to continue to assess these symptoms beyond the traditional 5-day period after cisplatin-based 
chemotherapy. Future studies should also consider assessing CINV beyond the traditional $120 \mathrm{~h}$ time point.

\section{CONCLUSION}

In conclusion, fosnetupitant appeared to be more effective than fosaprepitant in preventing CINV associated with cisplatin-based highly emetogenic chemotherapy during the extended 7-day period following chemotherapy in this exploratory analysis. When considering these findings in conjunction with the safety results, fosnetupitant may offer clinicians and patients a $\mathrm{NK}_{1}$ RA regimen with a better benefit-risk profile.

\section{ACKNOWLEDGEMENTS}

The authors are grateful to all the patients, their families, and the investigators who participated in this study.

Clinical Trial Information. A phase III, randomized, double-blind, multicenter, active control study of Pro-NETU for the prevention of chemotherapy induced nausea and vomiting (CINV) in patients receiving cisplatin-based highly emetogenic chemotherapy (HEC) (JapicCTI-194611); CONSOLE.

Funding. Sponsorship for this study was funded by Taiho Pharmaceutical Co., Ltd. The exploratory analysis was managed by Helsinn Healthcare and performed by Eros Papademetriou and Xing Liu at SmartAnalyst Inc. Support for the analytical assistance was funded by Helsinn Healthcare. The journal's Rapid Service Fee was also funded by Helsinn Healthcare.

Medical Writing, Editorial and Other Assistance. Medical writing and editorial assistance in the preparation of this article was provided by Jennifer Vanden Burgt, an independent medical writer. Support for the editorial assistance was funded by Helsinn Healthcare.
Authorship. All named authors meet the International Committee of Medical Journal Editors (ICMJE) criteria for authorship for this article, take responsibility for the integrity of the work as a whole, and have given their approval for this version to be published.

Author Contributions. Kaoru Kubota, Toshiaki Saeki, and Tomohide Tamura contributed to the primary study conception and design. Akito Hata, Yoshimasa Shiraishi, Naoki Inui, Morihito Okada, Masahiro Morise, Kohei Akiyoshi, Masayuki Takeda, Yasutaka Watanabe, Shunichi Sugawara, Naofumi Shinagawa, and Tomohide Tamura were involved in the collection of data. All authors contributed to the data interpretation, and critical review of the results and all versions of the manuscript.

Disclosures. Akito Hata: Consulting or Advisory Role: Boehringer Ingelheim, Lilly, Chugai Pharma, AstraZeneca, MSD. Speakers' Bureau: Boehringer Ingelheim, Lilly, Chugai Pharma, AstraZeneca, Taiho Pharmaceutical. Research Funding: Boehringer Ingelheim (Inst), MSD (Inst), Lilly (Inst), AstraZeneca (Inst). Yoshimasa Shiraishi: Honoraria: AstraZeneca, Lilly Japan, Chugai Pharma, Bristol Myers Squibb Company, Ono Pharmaceutical, Taiho Pharmaceutical. Research Funding: Chugai Pharma (Inst). Naoki Inui: Speakers' Bureau: Chugai Pharma, AstraZeneca, Lilly, Nippon Boehringer Ingelheim, Novartis, Ono Pharmaceutical, Taiho Pharmaceutical. Research Funding: Chugai Pharma, Daiichi Sankyo, Lilly, Nippon Boehringer Ingelheim, Taiho Pharmaceutical. Morihito Okada: Speakers' Bureau: Taiho Pharmaceutical, Johnson \& Johnson, Covidien, Lilly, Chugai Pharma, AstraZeneca, Ono Pharmaceutical, CSL Behring. Research Funding: Taiho Pharmaceutical (Inst), Nippon Kayaku (Inst), Chugai, Pharma (Inst), Covidien (Inst), Johnson \& Johnson (Inst), Daiichi Sankyo (inst), Yakult Honsha (Inst), Lilly Japan (Inst), Nihon Medi-Physics (Inst), Pfizer (Inst), Mochida Pharmaceutical Co, Ltd (Inst), Shionogi (Inst), Ono Pharmaceutical (Inst), Kyowa Hakko Kirin (Inst). Masahiro Morise: Speakers' Bureau: Chugai and Roche, AstraZeneca, Ono 
Pharmaceutical, Lilly. Research Funding: Boehringer Ingelheim (Inst), Novartis (Inst), AstraZeneca (Inst), Lilly (Inst), Taiho Pharmaceutical (Inst), Chugai and Roche (Inst), Ono. Pharmaceutical (Inst), Pfizer (Inst), Merck Serono (Inst), Kissei Pharmaceutical (Inst). Masayuki Takeda: Honoraria: AstraZeneca Japan, Chugai Pharma, Bristol Myers Squibb Company, Novartis, Ono Pharmaceutical, Boehringer Ingelheim. Shunichi Sugawara: Honoraria: AstraZeneca, Chugai Pharma, Nippon Boehringer Ingelheim, Taiho. Pharmaceutical, Pfizer, Lilly, Novartis, Bristol Myers Squibb, Ono. Pharmaceutical, MSD K K, Yakult Honsha, Kyowa Kirin. Naofumi Shinagawa: Speakers' Bureau: AstraZeneca, Novartis, Lilly, Merck Serono,Ono Pharmaceutical, Chugai Pharma, Boehringer Ingelheim. Research Funding: Olympus (Inst), Taiho Pharmaceutical (Inst), Boehringer Ingelheim (Inst), Lilly (Inst). Kaoru Kubota: Honoraria: Bristol Myers Squibb Japan, Daiichi Sankyo, Boehringer Ingelheim, Taiho Pharmaceutical, Lilly Japan, MSD Oncology, Chugai Pharma, AstraZeneca, Nihonkayaku, Takeda, Pfizer. Research Funding: Daiichi Sankyo (Inst), Boehringer Ingelheim (Inst), Taiho. Pharmaceutical (Inst), Ono Pharmaceutical (Inst). Toshiaki Saeki: Research Funding: Taiho Pharmaceutical (Inst), Eisai (Inst). Tomohide Tamura: Honoraria: Chugai Pharma, Boehringer Ingelheim, Ono Pharmaceutical, Taiho. Pharmaceutical, MSD, CMIC, Lilly, Nihonkayaku. There are no other potential conflicts of interest.

Compliance with Ethics Guidelines. This study was conducted according to the ethical principles of the Declaration of Helsinki and Good Clinical Practice guidelines and was conducted at all participating study sites after institutional review board approval. All patients provided written informed consent.

Data Availability. The Sponsor's policy on data sharing may be found at https://www. taiho.co.jp/en/science/policy/clinical_trial_ information_disclosure_policy/index.html.

Open Access. This article is licensed under a Creative Commons Attribution-NonCommercial 4.0 International License, which permits any non-commercial use, sharing, adaptation, distribution and reproduction in any medium or format, as long as you give appropriate credit to the original author(s) and the source, provide a link to the Creative Commons licence, and indicate if changes were made. The images or other third party material in this article are included in the article's Creative Commons licence, unless indicated otherwise in a credit line to the material. If material is not included in the article's Creative Commons licence and your intended use is not permitted by statutory regulation or exceeds the permitted use, you will need to obtain permission directly from the copyright holder. To view a copy of this licence, visit http://creativecommons.org/licenses/by$\mathrm{nc} / 4.0 /$.

\section{REFERENCES}

1. Navari RM, Aapro M. Antiemetic prophylaxis for chemotherapy-induced nausea and vomiting. N Engl J Med. 2016;374(14):1356-67.

2. Jordan K, Jahn F, Aapro M. Recent developments in the prevention of chemotherapy-induced nausea and vomiting (CINV): a comprehensive review. Ann Oncol. 2015;26(6):1081-90.

3. Kuchuk I, Bouganim N, Beusterien K, et al. Preference weights for chemotherapy side effects from the perspective of women with breast cancer. Breast Cancer Res Treat. 2013;142(1):101-7.

4. Sun CC, Bodurka DDC, Weaver CB, et al. Rankings and symptom assessments of side effects from chemotherapy: insights from experienced patients with ovarian cancer. Support Care Cancer. 2005;13(4):219-27.

5. Fernández-Ortega P, Caloto MT, Chirveches E, et al. Chemotherapy-induced nausea and vomiting in clinical practice: impact on patients' quality of life. Support Care Cancer. 2012;20(12):3141-8.

6. Van Laar ES, Desai JM, Jatoi A. Professional educational needs for chemotherapy-induced nausea and vomiting (CINV): multinational survey results from 2388 health care providers. Support Care Cancer. 2015;23(1):151-7.

7. ASCO 50th anniversary poll names top 5 advances past 50 years. The ASCO Post, October 15, 2014. 
8. Hesketh PJ. Chemotherapy-induced nausea and vomiting. N Engl J Med. 2008;358:2482-94.

9. Navari RM, Aapro M. Antiemetic prophylaxis for chemotherapy-induced nausea and vomiting. N Engl J Med. 2016;374:1356-67.

10. Hesketh PJ, Van Belle S, Aapro M, et al. Differential involvement of neurotransmitters through the time course of cisplatin-induced emesis as revealed by therapy with specific receptor antagonists. Eur J Cancer. 2003;39(8):1074-80.

11. Kris MG, Cubeddu LX, Gralla RJ, et al. Are more antiemetic trials with a placebo necessary? Report of patient data from randomized trials of placebo antiemetics with cisplatin. Cancer. 1996;78(10): 2193-8.

12. Bossi $\mathrm{P}$, Cortinovis D, Fatigoni S, et al. A randomized, double-blind, placebo-controlled, multicenter study of a ginger extract in the management of chemotherapy-induced nausea and vomiting (CINV) in patients receiving high-dose cisplatin. Ann Oncol. 2017;28(10):P2547-2551.

13. Pessi MA, Necchi A, Bossi P, et al. Nausea and vomiting during the first 3 intercycle periods in chemo-naive cancer patients receiving moderately/ highly emetogenic therapy. Tumori. 2015;101(6): 692-6.

14. Roila F, Molassiotis A, Herrstedt J, et al. Participants of the MASCC/ESMO consensus conference
Copenhagen 20152016 MASCC and ESMO guideline update for the prevention of chemotherapyand radiotherapy-induced nausea and vomiting and of nausea and vomiting in advanced cancer patients. Ann Oncol. 2016;27:v119-33.

15. Akynzeo ${ }^{\circledR}$ (netupitant and palonosetron) [prescribing information]. Lugano, Switzerland: Helsinn Therapeutics, Inc.; 2021.

16. Navari RM, Binder G, Bonizzoni E, et al. Single-dose netupitant/palonosetron versus 3-day aprepitant for preventing chemotherapy-induced nausea and vomiting: a pooled analysis. Future Oncol. 2021;17(23):3027-35.

17. Sugawara S, Inui N, Kanehara M, et al. Multicenter, placebo-controlled, double-blind, randomized study of fosnetupitant in combination with palonosetron for the prevention of chemotherapy-induced nausea and vomiting in patients receiving highly emetogenic chemotherapy. Cancer. 2019;125(22):4076-83.

18. Hata A, Okamoto I, Inui N, et al. Randomized, double-blind, phase III study of fosnetupitant versus fosaprepitant for prevention of highly emetogenic chemotherapy-induced nausea and vomiting: CONSOLE. J Clin Oncol. 2021. https://doi.org/10. 1200/JCO.21.01315.

19. Emend ${ }^{\circledR}$ (2021) (aprepitant) [prescribing information]. Merck \& Co, Whitehouse Station 